\title{
Laser-induced-fluorescence detection of nitric oxide in high-pressure flames with $A-X(0,2)$ excitation
}

\author{
Christof Schulz, Volker Sick, Johannes Heinze, and Winfried Stricker
}

\begin{abstract}
Laser-induced fluorescence techniques have been used successfully for quantitative two-dimensional measurements of nitric oxide. The commonly applied $D-X(0,1)$ or $A-X(0,0)$ schemes are restricted to atmospheric-pressure flames and engines driven with gaseous fuels because of strong attenuation of the exciting laser beam by combustion intermediates. The properties of a detection scheme for which excitation in the nitric oxide $A-X(0,2)$ band was used were investigated. We discuss the advantages of the $A-X(0,2)$ system (excited at $247.95 \mathrm{~nm}$ ) based on measurements in laminar premixed methane/air flames at 1-40 bars. (c) 1997 Optical Society of America

Key words: Laser-induced fluorescence, nitric oxide, high pressure, combustion.
\end{abstract}

\section{Introduction}

Detecting NO during the combustion process is of particular interest, as NO is one of the most important combustion-generated pollutants. Automotive engines especially play a major role in the overall production of this species that influences atmospheric chemistry in the respect of ozone depletion and smog formation. Drastic restrictions of NO release are expected in the following years. So despite the use of catalytic converters, it is an important task to make combustion cleaner.

Laser-induced fluorescence (LIF) is widely used to measure combustion-relevant species concentrations. ${ }^{1}$ Laser techniques provide the possibility of obtaining instantaneous two-dimensional images of absolute concentration fields without influencing the combustion process. Two-dimensional LIF measurements of minority species require the coincidence of strong electronic transitions with the emitted wavelength of intense light sources. Therefore excimer lasers are often used to match this requirement, and hence only a few excitation schemes are possible. For NO, different spectroscopic schemes are used.

C. Schulz and V. Sick are with the Physikalisch-Chemisches Institut, Universität Heidelberg, Im Neuenheimer Feld 253, 69120 Heidelberg, Germany. J. Heinze and W. Stricker are with the Institut für Physikalische Chemie der Verbrennung, DLR, Pfaffenwaldring 38-40, 70569 Stuttgart, Germany.

Received 26 August 1996; revised manuscript received 2 December 1996.

0003-6935/97/153227-06\$10.00/0

(C) 1997 Optical Society of America
The NO $D-X(0,1)$ system can be probed with $193-\mathrm{nm}$ radiation, e.g., from an $\mathrm{ArF}$ excimer laser. ${ }^{2}$ The $A-X(0,0)$ band at $225 \mathrm{~nm}$ can be excited with either a frequency-doubled dye laser ${ }^{3}$ or a $\mathrm{H}_{2}$ Raman-shifted $\mathrm{KrF}$ excimer laser. ${ }^{4}$

The NO $D-X(0,1)$ system at $193 \mathrm{~nm}$ is fine for clean atmospheric-pressure combustion processes in which no species that strongly absorb the exciting laser beam are present. So this method is usually restricted to combustion of $\mathrm{H}_{2}$ or small hydrocarbons at atmospheric pressure. Measurements in engines are reported ${ }^{5-7}$ but during an extended period of combustion, detection was not possible because of absorption of the incident laser beam. At high pressures, an additional interference with $\mathrm{O}_{2}$ must be expected. NO can no longer be excited selectively because of the broadened absorption lines of $\mathrm{O}_{2}$, which fluoresces efficiently in the range within which NO is detected. Furthermore, little is known about collisional quenching of the NO $D$ state under combustion conditions.

The NO $A-X(0,0)$ system at $225 \mathrm{~nm}$ has been applied successfully to measurements in highpressure $\mathrm{CH}_{4}$ air flames ${ }^{3}$ and in engines fueled with propane under conditions up to $20 \mathrm{~atm} .{ }^{8}$ Because of the large absorption cross sections and the high population of the vibrational ground level, relatively small laser-pulse energies ( $~ 5 \mathrm{~mJ})$ enabled measurements of two-dimensional concentration fields.

Nevertheless there are some disadvantages that suggest using longer wavelengths for excitation. At $248 \mathrm{~nm}$ the absorption is significantly reduced. ${ }^{9}$ The absorption of laser beams at different wavelengths was measured at a crank-angle position of $363^{\circ}$ (ignition at $340^{\circ}$ ) for an equivalence ratio of 1.0 in an engine run- 
ning at $1000 \mathrm{rpm}$ with propane fuel. Absorptions of $95 \% / \mathrm{cm}^{-1}$ at $193 \mathrm{~nm}, 25 \% / \mathrm{cm}^{-1}$ at $225 \mathrm{~nm}$, and less than $5 \% / \mathrm{cm}^{-1}$ at $248 \mathrm{~nm}$ were measured. Intermediate flame species (radicals, polycyclic aromatic, and partially oxidized hydrocarbons) formed during the combustion of larger aliphatic hydrocarbons (model fuels like $i$-octane or $n$-heptane) tend to absorb the 193and 225-nm radiation effectively. Additionally, the high NO concentration observed in engines can cause significant absorption of the laser beam at $225 \mathrm{~nm}$. The small Boltzmann fraction in $v^{\prime \prime}=2$ results in a small absorption cross section at $248 \mathrm{~nm}$ and thus negligible absorption.

We discuss the spectroscopic properties of $\mathrm{NO}$ compared with those of $\mathrm{O}_{2}$ as the major interfering species in the NO $A-X(0,2)$ bandhead region near 248 $\mathrm{nm}$. Both must be known to enable quantitative measurements and to provide an excitation detection scheme for which interferences can be neglected:

$$
I \propto I_{L} N(p, T) f_{B} B_{k i} g_{\lambda}(p, T) \frac{A_{i k}}{A_{k}+P+Q(p)} .
$$

The LIF intensity $I$ for one species (in the linear regime, neglecting rotational and vibrational energy transfers) is proportional to the incident laser intensity $I_{L}$ and depends on the number density of the excitable molecules [which is the number density $N(p, T)$ of the species times the Boltzmann fraction $f_{B}$ of the initial level), the Einstein $B_{k i}$ coefficient for the absorption, the spectral overlap $g_{\lambda}(p, T)$ of the laser profile and the absorption line, and the fluorescence quantum yield. The latter depends on the Einstein $A_{i k}$ coefficient for the emission divided by the sum of the rates of all depopulation processes of the excited state [spontaneous emission $A_{i k}$, predissociation $P$, and quenching $Q(p)]$. The Einstein coefficients can be calculated from spectroscopic data. ${ }^{10}$ Broadband detection provides the detection of all rotational transitions. Thus the explicit implementation of rotational energy transfer and filter functions was omitted in expression (1). In the discussion below about the interference with $\mathrm{O}_{2}$ fluorescence, this topic is addressed in more detail.

For NO at atmospheric pressure and above, the denominator in expression (1) is dominated by electronic quenching $Q(p)$. The predissociation rate $P$ is negligible for the transitions considered here, and $A$ $\ll Q(p)$. The total quenching rates scale linearly with the pressure. Quenching data for a variety of species are available for the $v^{\prime}=0$ state. ${ }^{11,12}$ It was shown that the spatial variation of $Q(p)$ is less than $10 \%$ for typical engine conditions. ${ }^{8,12}$

Both the fluorescence quenching $Q(p)$ and the number density $N$ increase linearly with the pressure. Hence for NO, expression (1) can be transformed into expression (2), in which $g_{\lambda}(p, T)$ is the only remaining pressure-dependent factor:

$$
I \propto I_{L} N(p, T) f_{B} B_{k i} g_{\lambda}(p, T) \frac{A_{i k}}{q}
$$

where $q=Q(p) / p$. Pressure broadening and shifting of the rotational fine structure change the overlap with the laser line profile. ${ }^{13,14}$ We used the coefficients given in Ref. 14 for $\mathrm{N}_{2}$ as representatives for line-broadening and line-center shifts. Details of the spectral simulations will be discussed in a forthcoming paper.

Significant amounts of NO are formed in flames mainly above $1700 \mathrm{~K}$. At combustion temperatures, the variation of the ground-state population in the second vibrational level is only $\sim 5 \%$ per $100 \mathrm{~K}$ (near $2200 \mathrm{~K}$ ) for the rotational levels included in the $\mathrm{O}_{12}$ bandhead region at $247.95 \mathrm{~nm}$. The variation decreases for increasing temperatures. Thus semiquantitative measurements of NO distributions are possible without detailed knowledge of the local temperature. If simultaneous measurements of temperature fields are performed, ${ }^{9}$ a correction to the local temperature is possible.

For $\mathrm{O}_{2}$ the denominator of expression (1) is dominated by the predissociation rate $P$ up to at least 10 bars for the transitions considered here. ${ }^{15}$ For this pressure range, the fluorescence quantum yield is pressure independent and the $\mathrm{O}_{2}$ signal rises linearly with the increasing number density. So for NO measurements in high-pressure environments, it is important to prevent the detection of $\mathrm{O}_{2}$ carefully.

Under high-pressure conditions Raman, scattering (especially from $\mathrm{N}_{2}$ and $\mathrm{H}_{2} \mathrm{O}$ ) becomes an important interference too. The Raman signal intensity per unit mole fraction increases linearly with pressure, whereas the NO LIF signal decreases because of less overlap between the laser line and the pressurebroadened absorption line, as described with the factor $g_{\lambda}(p, T)$ in expression (1).

Because the transition used for NO detection starts in a higher vibrational level $\left(v^{\prime \prime}=2\right)$, the ground-state population is small even at flame temperatures and LIF signals will be weak despite the large Einstein $B$ coefficients of the $(0,2)$ band. So intense light sources are obligatory. The NO $A-X(0,2) \mathrm{O}_{12}$ bandhead can be excited at the short-wavelength end of the tuning range of a commercial $\mathrm{KrF}$ excimer laser where the spectral purity is still excellent. For shorter wavelengths the locking efficiency drops significantly and the increasing energy of broadband amplified spontaneous emission disables selective excitation. We succeeded in extending the tuning range down to 247.5 $\mathrm{nm}$ by using the $\mathrm{KrF}$ excimer laser as an amplifier for frequency-doubled dye laser light. ${ }^{16}$ Nevertheless the bandhead region at $247.95 \mathrm{~nm}$ can be excited selectively with both laser systems. They provide laser energies in excess of $100 \mathrm{~mJ}$ per pulse. Planar imaging experiments exciting the weakly populated $v^{\prime \prime}=2$ level are thus possible when this transition is used.

\section{Experimental}

Laminar, premixed $\mathrm{CH}_{4}$ /air flames at pressures between 1 and 40 bars were stabilized on a sintered bronze plate. ${ }^{17}$ This 8 -mm-diameter plate was mounted in a stainless steel housing with an inner diameter of $60 \mathrm{~mm}$. The pressure could be stabilized 


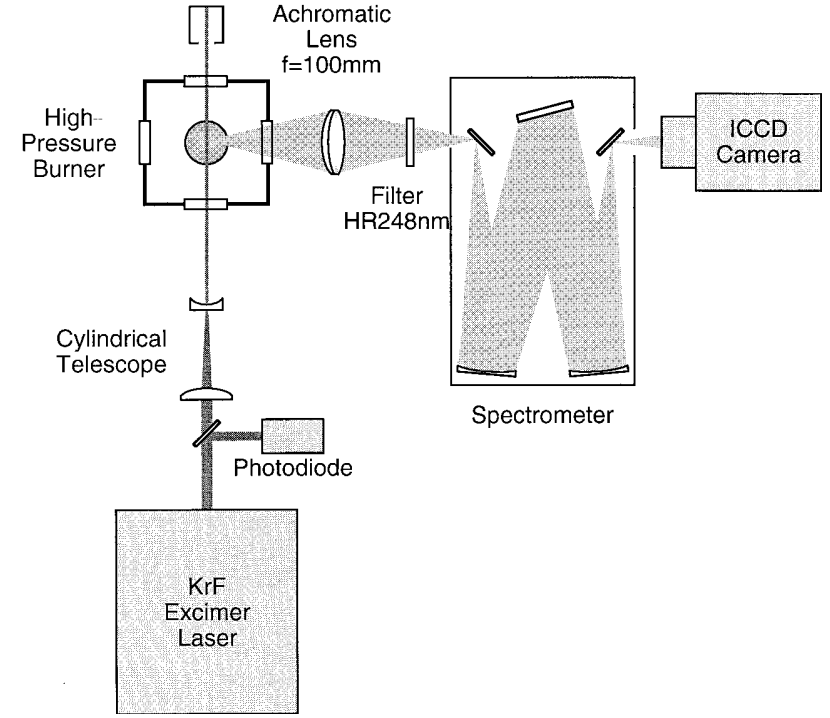

Fig. 1. Experimental setup for spectroscopic measurements on $\mathrm{NO}$ in a lean $(\phi=0.9)$, premixed $\mathrm{CH}_{4} /$ air flame operated at $1-40$ bars. In the measurements presented here, $370 \mathrm{ppm}$ of $\mathrm{NO}$ were doped to the burn gases (see text).

to \pm 0.03 bar. For all data presented here, the equivalence ratio was fixed at $\phi=0.9$, which results in NO concentrations of the order of 50 parts in $10^{6}(\mathrm{ppm})$. To increase the signal-to-noise ratio, typically 370 ppm of NO were doped to the gas mixture, which is still much lower than NO concentrations found in engine measurements. ${ }^{18}$ Optical access to the flame was possible by means of four quartz windows (Fig. 1). The beam of a tunable $\mathrm{KrF}$ excimer laser (Lambda Physik EMG 150 TMSC, FWHM $0.5 \mathrm{~cm}^{-1}$ ) was aligned parallel to the burner surface that passed the flame through the center. Alternatively, the first anti-Stokes line from a $\mathrm{H}_{2}$ (10-bars) Raman shifter was used. The pulse energy was measured with a fast photodiode. Focusing the beam with a cylindrical telescope to form a light sheet with a $20 \mathrm{~mm} \times 0.5$ $\mathrm{mm}$ cross section yielded spectral intensities of 40 $\mathrm{MW} / \mathrm{cm}^{2} \mathrm{~cm}^{-1}$ at $247.95 \mathrm{~nm}$ and $1.3 \mathrm{MW} / \mathrm{cm}^{2} \mathrm{~cm}^{-1}$ at $225.25 \mathrm{~nm}$. The fluorescence signals were collected at right angles to the laser beam and focused with a $f=100 \mathrm{~mm}, f \#=2$ achromatic lens onto the entrance slit of a 500-mm imaging spectrometer (Chromex 500IS) that was equipped with a 300grooves $/ \mathrm{mm}$ grating operated in second order. Locking efficiencies were measured by Rayleigh scattering with a 2400 -grooves/mm grating. Emission spectra were recorded in two independent runs from 225 to 270 and from 270 to $310 \mathrm{~nm}$. A narrow-band dielectric mirror (high reflectivity of $248 \mathrm{~nm}, 0^{\circ}$, FWHM $10 \mathrm{~nm}$, Laser Optik) was used as a filter to reduce the intensity of the Rayleigh signal by 2 orders of magnitude. The dispersed fluorescence signals were detected with an intensified CCD camera (LaVision FlameStar III). Each laser pulse yielded a complete emission spectrum with additional spatial resolution in the direction of the spectrometer slit. The signal was averaged over 250

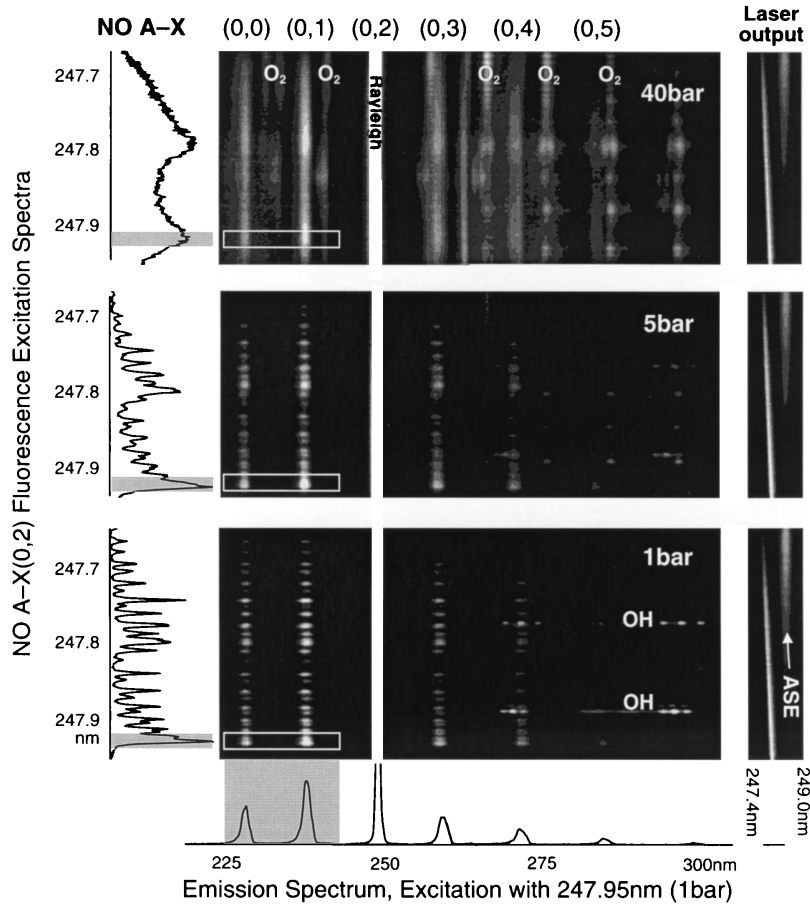

Fig. 2. Excitation-emission spectra obtained in the lean $\mathrm{CH}_{4} /$ air flame at 1,5 , and 40 bars. The spectra on the left show profiles along the NO $A-X(0,1)$ emission lines for excitation wavelengths between 247.65 and $248.0 \mathrm{~nm}$ at 1,5 , and 40 bars. The spectra are not corrected for wavelength dependence of the laser energy. The emission spectrum at the bottom was obtained at 1 bar after excitation at $247.95 \mathrm{~nm}$. The NO $A-X\left(0, v^{\prime \prime}\right)$ progression is clearly resolved with the $(0,2)$ band overlapped with the Rayleigh peak (off scale). The small frames at the right show enlarged excitationemission spectra of the laser output obtained by Rayleigh scattering. The intensity of the narrow-band part (diagonal line) drops significantly at wavelengths shorter than $247.8 \mathrm{~nm}$. ASE, amplified spontaneous emission.

single shots. When excitation-emission spectra were recorded, the spectrally resolved signal was averaged over the spatial coordinate and stored before the laser was scanned to the next spectral position (1 step: $0.18 \mathrm{~cm}^{-1}$ ). With this procedure two-dimensional maps with excitation and emission frequency as coordinates are recorded. Taking profiles along the excitation-frequency coordinate yields excitation spectra, for which the bandpass of the detection can be arbitrarily set to a desired value within the entire emission coordinate. Similarly, emission spectra can be extracted with the excitation frequency's being fixed at a given value.

\section{Results and Discussion}

It has been shown in recent years that tunable $\mathrm{KrF}$ excimer lasers can be used to detect $\mathrm{OH}, \mathrm{O}_{2}$, and $\mathrm{H}_{2} \mathrm{O}$ with LIF. ${ }^{19}$ A detection scheme for detecting NO with these lasers has to minimize interference with any of these molecules.

Two-dimensional excitation-emission spectra, as shown in Fig. 2, give an overview of all fluorescing species and allow us to compare their contribution with a total fluorescence intensity. Cuts along hor- 
izontal lines show emission spectra after excitation at different wavelengths, whereas vertical lines represent fluorescence excitation spectra. The spectra are not corrected for the decreasing laser energy at the lower end of the tuning range of the $\mathrm{KrF}$ excimer laser. Thus they allow us to compare the LIF intensities that can be obtained with this laser system. At wavelengths shorter than $247.75 \mathrm{~nm}$ there is a significant amount of broadband amplified spontaneous emission that causes unstructured fluorescence by $\mathrm{O}_{2}$ (see right-hand side of Fig. 2).

Detection schemes suitable for two-dimensional imaging experiments must match different requirements: They should enable a selective detection of one species, and the transmission of the filtering system should be high enough to provide sufficiently high signal intensities to enable single-shot measurements. We use relatively broad bandpass filters (FWHM $15 \mathrm{~nm}$ ) for our imaging experiments. The filters are composed of four $45^{\circ}$ mirrors, coated to reflect only the desired signal wavelength band. Using four of these mirrors in series results in a steep filter function, which is efficient for rejecting the nearby excitation wavelength. Additionally, a normal-incidence mirror coated for $248 \mathrm{~nm}$ was placed in front of the filter to block Rayleigh and Mie scattering. To minimize interferences with other species, excitation and detection wavelengths have to be chosen carefully.

In Fig. 2, detection schemes are represented by rectangles with the height of the laser bandwidth and the width of the detection filter function. Exciting the $\mathrm{NO} \mathrm{O}_{12}$ bandhead at $247.95 \mathrm{~nm}$ and detecting the anti-Stokes emissions between 220 and $240 \mathrm{~nm}$ provide a fluorescence signal that is by far dominated by NO.

Pressure broadening causes blending of the rotational lines with increasing pressure. Although the rotational structure is smeared out at 40 bars, the scheme allows a selective detection of NO.

It can be seen (Fig. 2) that the intensity of $\mathrm{OH}$ $A-X\left(3, v^{\prime \prime}\right)$ decreases with increasing pressure. Because of the anti-Stokes detection of NO, no interferences with $\mathrm{OH}$ LIF are expected anyway. $\mathrm{H}_{2} \mathrm{O}$ would be excited by a two-photon transition, and subsequent fluorescence from the $\mathrm{OH}$ fragment would be outside the NO detection bandpass.

Hence, mainly $\mathrm{O}_{2}$, which in lean and stoichiometric flames is present in relatively high concentrations, must be considered. Di Rosa et al. ${ }^{20}$ suggest a simultaneous measurement of $\mathrm{O}_{2} \mathrm{LIF}$ between 300 and 400 $\mathrm{nm}$ and subtraction from the overlapped $\mathrm{NO}$ and $\mathrm{O}_{2}$ signals. In the combustion of hydrocarbons, however, strong LIF signals from hydrocarbons and combustion intermediates are observed in this wavelength range. ${ }^{21}$ Thus no selective measurement of the $\mathrm{O}_{2}$ background is possible in this environment.

All spectra shown here were taken in a lean flame $(\phi$ $=0.9)$ to make the $\mathrm{O}_{2}$ signal prominent. The $\mathrm{O}_{2}$ $B-X(0,6)$ band appears with strong rotational transitions at emission wavelengths longer than $255 \mathrm{~nm}$. Because of the small Einstein coefficients for the $(0,5)$

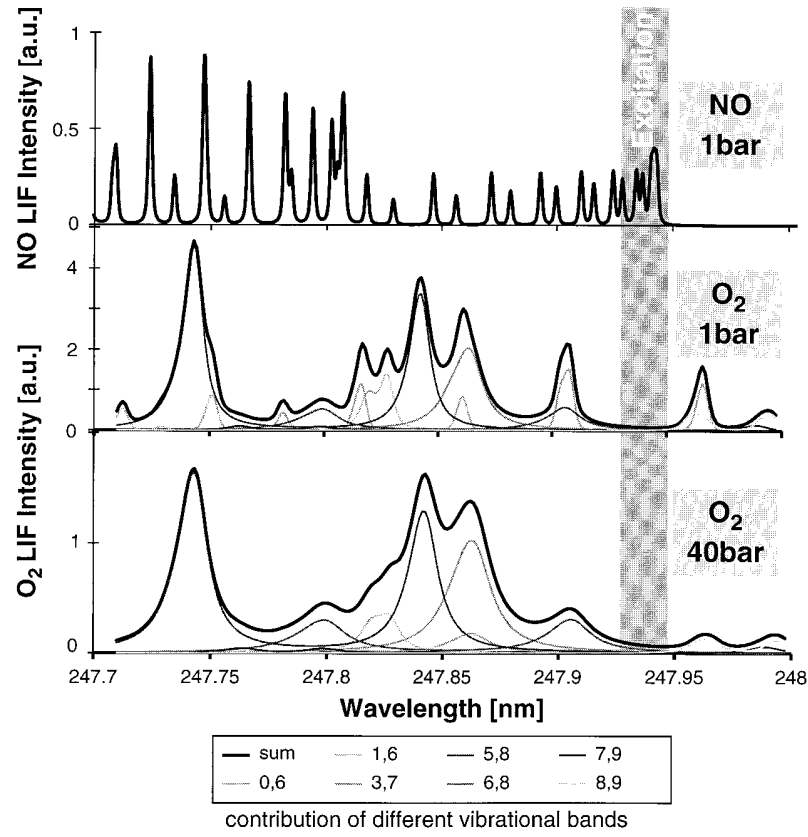

Fig. 3. Comparison of calculated fluorescence excitation spectra of $\mathrm{NO}$ and $\mathrm{O}_{2}$ near the NO $A-X(0,2) \mathrm{O}_{12}$ bandhead at 1 and 40 bars. Only the $\mathrm{O}_{2}$ signal that is emitted within the experimental bandpass suggested for NO detection is taken into account.

and the $(0,4)$ bands, these transitions do not affect the detected interval between 220 and $240 \mathrm{~nm}$. Because of rapid predissociation, fluorescence quenching and pressure broadening are negligible up to at least 10 bars. ${ }^{15}$ Hence the LIF intensity of $\mathrm{O}_{2}$ increases significantly with pressure. Figure 2 shows that the strongest $\mathrm{O}_{2}$ emissions appear to be Stokes shifted but at pressures greater than 10 bars there are contributions of the $\mathrm{O}_{2}$ LIF signal in the interval between 220 and $240 \mathrm{~nm}$ too. This signal, with a maximum at excitation wavelengths near $247.85 \mathrm{~nm}$, is caused mainly by $\mathrm{O}_{2} B-X(5,8)$ and $(3,7)$ transitions. Figure 3 shows simulations of $\mathrm{O}_{2}$ fluorescence spectra for pressures up to 40 bars, assuming detection between 220 and $240 \mathrm{~nm}$. It can be seen that the NO bandhead region matches a minimum in the $\mathrm{O}_{2}$ fluorescence spectrum; thus the interference of $\mathrm{O}_{2}$ is minimized.

For comparison, Fig. 4 shows excitation-emission spectra taken with the Raman-shifted $\mathrm{KrF}$ excimer laser. A section of the frequently used NO $A-X(0,0)$ band near $225.2 \mathrm{~nm}$ is shown. Excitation of the $R_{1}$ 21.5 rotational transition and detection of the NO $A-X(0,2)$ emission at $847 \mathrm{~nm}$ allows a selective measurement of NO up to 20 bars. At higher pressures, pressure-broadened $\mathrm{O}_{2}$ fluorescence appears in the lean flame that can no longer be suppressed with bandpass filters. Nevertheless, at high pressures this scheme is suited for one-dimensional measurements along a line when the fluorescence light is spectrally resolved with a monochromator.

Figure 5 presents the change of NO excitation spectra near $248 \mathrm{~nm}$ as a function of pressure. Shown are measured spectra compared with calculations. The 


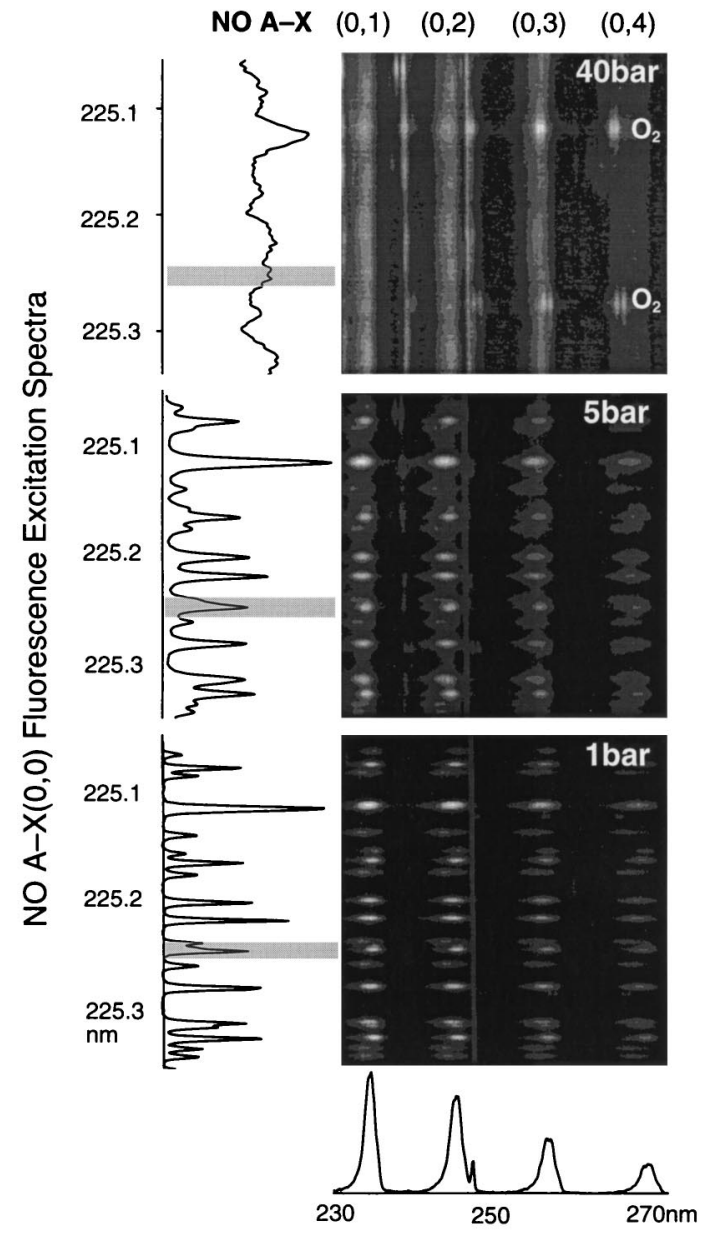

Emission Spectrum, Excitation with 225.25nm (1bar)

Fig. 4. Excitation-emission spectra obtained in the lean methane/air flame at 1, 5, and 40 bars for excitation between 225.05 and $225.35 \mathrm{~nm}$ using the Raman-shifted $\mathrm{KrF}$ excimer laser.

pressure-broadening coefficient that is extracted from the data is in agreement with the coefficient published by Di Rosa and Hanson. ${ }^{14}$ No experimental evidence for saturation was found. Thus the measured spectra were corrected for the energy of the narrow-band part of the laser emission obtained from highly resolved two-dimensional excitation-emission plots of Rayleigh signals (see Fig. 2). The good agreement between measurement and simulation enables the calculation of the pressure-dependent factor $g_{\lambda}(p, T)$ as a convolution between the laser line shape and the LIF spectrum. Figure 6 shows these overlap coefficients for several spectral laser linewidths. It is obvious from Fig. 6 that a narrow-band laser is not desirable for the measurements if one wishes to avoid strong corrections. However, one has to balance between a pressure-independent excitation efficiency and possible interferences with fluorescence from $\mathrm{O}_{2}$ (see Fig. 3).

The detection at shorter wavelengths relative to the excitation wavelength avoids problems with interference that is due to Raman signals, which become an increasing problem with increasing pressure. Although NO LIF is more or less pressure

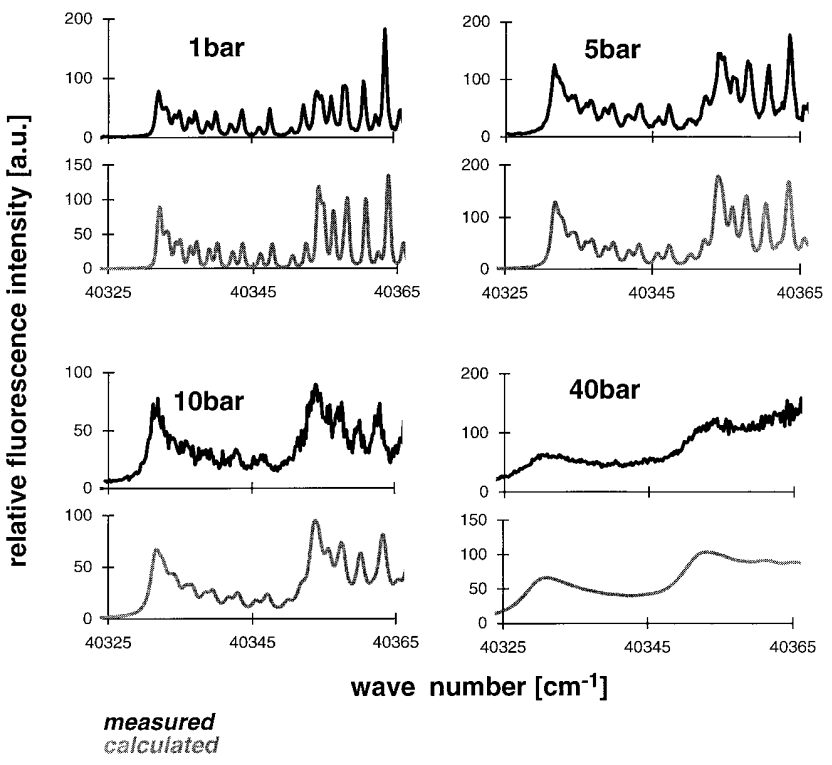

Fig. 5. Measured and calculated fluorescence excitation spectra near the NO $A-X(0,2) \mathrm{O}_{12}$ bandhead for different pressures. The measured spectra are corrected for changes in laser intensity.

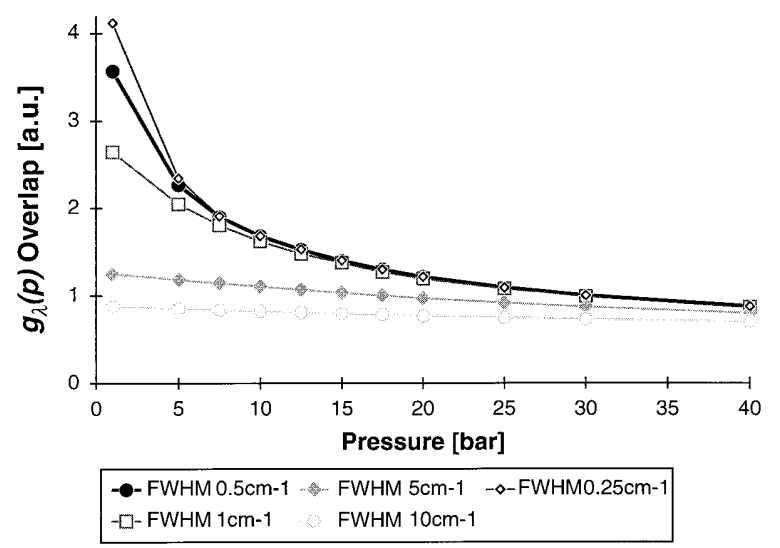

Fig. 6. Pressure dependence of spectral overlap $g_{\lambda}(p, T)$ of the NO fluorescence excitation spectra and the laser line shape that is assumed to be Gaussian. The calculations are done for $T=2000 \mathrm{~K}$.

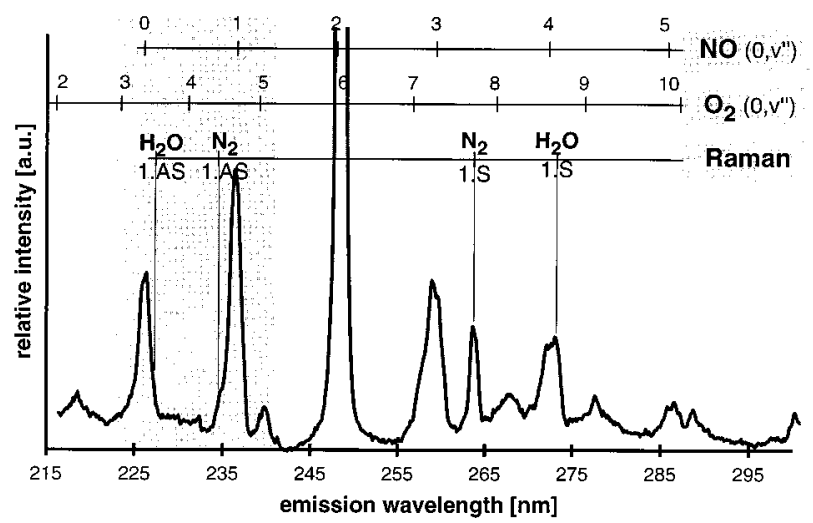

Fig. 7. Emission spectrum at 40 bars after excitation at 247.95 $\mathrm{nm}$. The shaded area marks the detection bandpass. 
independent (see discussion above), the Raman signal increases linearly with pressure. Figure 7 shows an emission spectrum at 40 bars after excitation at $247.95 \mathrm{~nm}$. Although the Raman-Stokes lines of $\mathrm{N}_{2}$ and $\mathrm{H}_{2} \mathrm{O}$ are a significant portion of the emissions at longer wavelengths, their influence on the anti-Stokes side is much smaller. The intensity ratio of the anti-Stokes over the Stokes signal is $\sim 0.18$ for $\mathrm{N}_{2}$ and 0.07 for $\mathrm{H}_{2} \mathrm{O}$ at $2000 \mathrm{~K}$, deduced from the Boltzmann population of the first vibrational levels. Hence a NO detection scheme in the anti-Stokes regime minimizes the influence of Raman scattering by at least a factor of 5 .

\section{Conclusions}

The new NO $A-X(0,2)$ detection scheme with excitation of the $\mathrm{NO} \mathrm{O}_{12}$ bandhead at $247.95 \mathrm{~nm}$ and detection of the $(0,0)$ and the $(0,1)$ bands between 220 and $240 \mathrm{~nm}$ is suited to measure quantitative concentrations of NO selectively in combustion processes under high-pressure conditions. Measurements in lean high-pressure flames up to 40 bars doped with 370 ppm of NO show that the signal is clearly dominated by NO fluorescence for all spectra. Raman, Rayleigh, and LIF signals from other species, mainly $\mathrm{O}_{2}$, can be suppressed effectively. The high pulse energy of tunable $\mathrm{KrF}$ excimer lasers provides pulse energies high enough for two-dimensional single-shot imaging while still remaining in the linear excitation regime. The ability and characteristics for two-dimensional singleshot measurements in a transparent spark ignition engine fueled with isooctane was shown elsewhere. ${ }^{9}$ A more detailed experimental and numerical investigation of saturation effects is in progress.

The authors thank M. Decker (DLR, Stuttgart) for assistance during the measurement and helpful discussions and J. Wolfrum (Physikalisch-Chemisches Institut, Heidelburg) for his continuous motivation of this work. This work has been funded by the Bundesministerium für Bildung, Forschung, und Technologie under contract 13N6283 (PhysikalischChemisches Institut, University of Heidelberg) and 13N6119 (DLR Stuttgart).

\section{References}

1. A. C. Eckbreth, Laser Diagnostics for Combustion, Temperature and Species (Abacus, Turnbridge Wells, England, 1988); K. Kohse-Höinghaus, "Laser techniques for the quantitative detection of reactive intermediates in combustion systems," Prog. Energy. Combust. Sci. 20, 203-247 (1994).

2. A. M. Wodtke, L. Huwel, H. Schlüter, G. Meijer, P. Andresen, and H. Voges, "High sensitivity detection of NO in a flame using a tunable ArF laser," Opt. Lett. 13, 910-912 (1988).

3. A. O. Vyrodow, J. Heinze, M. Dillmann, U. E. Meier, and W. Stricker, "Laser-induced fluorescence thermometry and concentration measurements on NO $A-X(0,0)$ transitions in the exhaust gas of high pressure $\mathrm{CH}_{4}$ /air flames," Appl. Phys. B 61, 409-414 (1995).

4. T. Dreier, A. Dreizler, and J. Wolfrum, "The application of a Raman-shifted tunable $\mathrm{KrF}$-excimer laser for laser induced fluorescence combustion diagnostics," Appl. Phys. B 55, 381387 (1992).
5. P. Andresen, G. Meijer, H. Schlüter, H. Voges, A. Koch, W. Hentschel, W. Oppermann, and E. W. Rothe, "Fluorescence imaging inside an internal combustion engine using tunable excimer lasers," Appl. Opt. 29, 2392-2404 (1990).

6. A. Arnold, F. Dinkelacker, T. Heitzmann, P. Monkhouse, M. Schäfer, V. Sick, J. Wolfrum, W. Hentschel, and K. P. Schindler, "DI Diesel engine combustion visualized by combined laser techniques", in Proceedings of the Twenty-Fourth International Symposium on Combustion (The Combustion Institute, Pittsburgh, Pa., 1992), pp. 1605-1612.

7. Th. M. Brugman, R. Klein-Douwel, G. Huigen, E. van Walwijk, and J. J. ter Meulen, "Laser induced fluorescence imaging of NO in an $n$-heptane and diesel fuel driven diesel engine," Appl. Phys. B 57, 405-410 (1993).

8. A. Bräumer, V. Sick, J. Wolfrum, V. Drewes, R. R. Maly, and M. Zahn, "Quantitative two-dimensional measurements of nitric oxide and temperature distributions in a transparent SI engine," in 1995 SAE Congress and Exposition (Society of Automotive Engineers, Warrendale, Pa., 1995), paper 952462.

9. C. Schulz, V. Sick, J. Wolfrum, V. Drewes, M. Zahn, and R. Maly, "Quantitative 2D single-shot imaging and mathematical modeling of NO concentrations and temperatures in a transparent SI engine," in Proceedings of the Twenty-Sixth International Symposium on Combustion (The Combustion Institute, Pittsburgh, Pa., 1996), pp. 2597-2604.

10. C. O. Laux and C. H. Kruger, "Arrays of radiative transition probabilities for the $\mathrm{N}_{2}$ first and second positive, NO beta and gamma, $\mathrm{N}_{2}{ }^{+}$first negative, and $\mathrm{O}_{2}$ Schumann-Runge band systems," J. Quant. Spectrosc. Radiat. Transfer 48, 9-24 (1992).

11. P. H. Paul, J. A. Gray, J. L. Durant Jr., and J. W. Thoman Jr., "A model for temperature-dependent collisional quenching of NO $A^{2} \Sigma^{+}$," Appl. Phys. B 57, 249-259 (1993).

12. P. H. Paul, J. A. Gray, J. L. Durant Jr., and J. W. Thoman Jr., "Collisional quenching corrections for laser induced fluorescence measurements of NO $A^{2} \Sigma^{+}$," AIAA J. 32, 1670-1675 (1994).

13. M. D. Di Rosa and R. K. Hanson, "Collision-broadening and -shift of $\mathrm{NO} \gamma(0,0)$ absorption lines by $\mathrm{H}_{2} \mathrm{O}, \mathrm{O}_{2}$ and $\mathrm{NO}$ at 295K," J. Mol. Spectrosc. 164, 97-117 (1994).

14. M. D. Di Rosa and R. K. Hanson, "Collisional broadening and shift of $\mathrm{NO} \gamma(0,0)$ absorption lines by $\mathrm{O}_{2}$ and $\mathrm{H}_{2} \mathrm{O}$ at high temperatures," J. Quant. Spectrosc. Radiat. Transfer 52, 515529 (1994).

15. V. Sick, M. Decker, J. Heinze, and W. Stricker, "Collisional processes in the $\mathrm{O}_{2} B^{3} \Sigma_{u}{ }^{-}$state," Chem. Phys. Lett. 249, 335-340 (1996).

16. C. Schulz, B. Yip, V. Sick, and J. Wolfrum, "A laser-induced fluorescence scheme for measuring nitric oxide in engines," Chem. Phys. Lett. 242, 259-264 (1995).

17. H. Eberius, T. Just, T. Kick, G. Häfner, and W. Lutz, "Stabilization of premixed, laminar methane flames in the pressure regime up to 40 bar," Proceedings of the Joint Meeting of the German/Italian Section of the Combustion Institute (The Combustion Institute, Pittsburgh, Pa., 1989), p. 3.3.

18. J. B. Heywood, Internal Combustion Engine Fundamentals (McGraw-Hill, New York, 1988), pp. 578-586.

19. P. Andresen, A. Bath, W. Gröger, H. W. Lülf, G. Meijer, and J. J. ter Meulen, "Laser-induced fluorescence with tunable excimer lasers as a possible method for instantaneous temperature field measurements at high pressures: checks with an atmospheric flame," Appl. Opt. 26, 365 (1988).

20. M. D. Di Rosa, K. G. Klavuhn, and R. K. Hanson, "LIF spectroscopy of $\mathrm{NO}$ and $\mathrm{O}_{2}$ in high-pressure flames," in Proceedings of the 1995 Fall Meeting of the Western States Section of the Combustion Institute (The Combustion Institute, Pittsburgh, Pa., 1995).

21. A. Arnold, W. Ketterle, H. Becker, and J. Wolfrum, "Simultaneous single-shot imaging of $\mathrm{OH}$ and $\mathrm{O}_{2}$ using a twowavelength excimer laser," Appl. Phys. B 51, 99-102 (1990). 